The Japanese Journal of Animal Psychology, 50, 1, 27-31 (2000)

Short Report

\title{
Classical backward conditioning in second-order conditioning in rat's conditioned suppression
}

\author{
KOUJI URUSHIHARA ${ }^{1}$ \\ Kwansei Gakuin University
}

\begin{abstract}
Several studies showed the second order conditioning with a first order backward conditioned stimulus as a reinforcer established an excitatory conditioned response to the second order conditioned stimulus, while no excitation to the first order conditioned stimulus being observed. But other previous studies failed to show the similar effects in other situations. The present experiment addressed to retest the effects in the rats' licking suppression, in which a great part of procedures was similar to those used in the successful previous studies, except with on-the-baseline technique that had never been adopted in any previous studies. As the result, the second order excitation was observed but no eminently first order excitation was seen; i.e., the second order excitation with first order backward conditioning could be successfully demonstrated.
\end{abstract}

Key words : cassical conditioning, backward conditioning, second order conditioning, conditioned suppression, rat.

Pavlov (1927) found that the classical backward conditioning procedure (i.e., an unconditioned stimulus, US, followed by an conditioned stimulus, CS) established no conditioned response, CR. The result was interpreted as a lack of an association between the CS and the US at the time. But recently, several studies indicated that an excitatory association could be formed as the result of the backward conditioning procedure, though no eminently CR was observed (Barnet, Arnold, \& Miller, 1991; Barnet \& Miler, 1996; Cole \& Miller, 1999; Matzel, Held, \& Miller, 1988). These studies employed a kind of higher order conditioning paradigm such as the second order conditioning or the sensory preconditioning. They observed no CR to the CS1, which was paired directly with the US, but observed a significant CR to the CS2, which was paired not with the US but with the CS1. That is, the backward conditioning procedure endows the CS with little power of eliciting $\mathrm{CR}$, but enough power of forming an excitatory higher order conditioning (see

1 Reprint request e-mail : uru@pluto.dti.ne.jp
Urushihara, 1999; Urushihara \& Nakajima, 1999, for further discussion).

These findings, however, are observed only in the limited laboratory; the double checks in other laboratories failed to show the similar effects (Hemmes, Brown, \& Vaca, 1994; Stern \& Frey, 1978). In particular, Urushihara and Imada (1999) failed to show the second order excitation with first order backward conditioning, in spite that they employed the same conditioning preparation, rat's conditioned suppression of licking, as that used in the previous successful studies, whereas they observed the second order excitation with first order forward conditioning in the same experiment. This finding may cast doubt on robustness of the phenomenon.

But recently, Cole and Miller (1999) showed interesting data. They examined the effect of number of first order backward conditioning trials on the second order excitation. As a result, they found that the second order excitation was established when the number of first order backward conditioning trials was large enough to elicit inhibitory first order backward conditioning, but the second order excitation was 
never observed with moderate number of trials, with which neither excitatory nor inhibitory first order backward conditioning was observed. This result suggests the possibility that the trial number employed in Urushihara and Imada (1999) was not suitable for forming the second order excitation with backward first order conditioning. The first goal of the present experiment is to retest the phenomenon of the second order excitation with first order backward conditioning with more number of first order backward conditioning trials than in Urushihara and Imada.

The present experiment was run in rats' licking suppression, a similar preparation to those employed in the previous studies showing the second order excitation with first order backward conditioning, and its procedure was almost identical to that used in Urushihara and Imada (1999). In all of the previous studies showing the second order excitation with first order backward conditioning, an off-the-baseline procedure was employed. As the result, the process of forming the second order excitation could not be observed. The effects were shown only in probe tests, which was conducted after conditioning procedures had finished at all. The second goal of the present experiment is to observe the process of forming the second order excitation with first order backward conditioning employing on-the-baseline procedure.

\section{Method}

\section{Subjects}

Thirty-six experimentally naive male Wistar rats (Rattus norvegicus) were used. They were approximately 10 weeks old at the beginning of the experiment. During experimental sessions, food was always available, but water was available for only $15 \mathrm{~min}$. after each experimental session.

\section{Apparatus}

Six drinking boxes, measuring $20 \mathrm{~cm}$ long, 10 $\mathrm{cm}$ wide, and $15 \mathrm{~cm}$ high, were located in a radial position on a table of the experimental room. Each box was made of white acrylic walls, a clear acrylic ceiling, and a grid floor consisting of 5-mm metal rods spaced $13 \mathrm{~mm}$ center to center. One narrow wall, the front panel, had a hole, $12 \mathrm{~mm}$ in diameter. It was in right-left center of the front panel and $45 \mathrm{~mm}$ above the grid floor. Subjects could access a drinking spout through this hole. Contact with the spout was detected by a low-current circuit between the spout and the floor. A 90-W lamp fixed in a lampshade was suspended $1.6 \mathrm{~m}$ above the boxes, and a speaker was located $0.5 \mathrm{~m}$ above them. A 0.7 -s scrambled electric shock $(0.86 \mathrm{~mA}$, assuming the resistance of a subject as $100 \mathrm{k} \Omega$ )was used as an US.

The color of the walls was able to be changed to black by equipping black plastic plates inside the box. When they were equipped, the hole was covered and the drinking spout was not accessible. Thus, the box had two different contexts, one was a white context (CTX-W) and the other was a black context (CTX-B). The boxes were illuminated $(2.2 \mathrm{~lx}$ ) by an indirect lighting of room lamps when used the CTX-W; no illumination was done when used the CTX-B $(0.0$ lx). All events were controlled and recorded by a computer located in an adjacent room.

\section{Stimuli}

The second order conditioned stimulus (CS2) was turning on a $90-\mathrm{W} \operatorname{lamp}(8.3 \mathrm{~lx})$ for all subjects. The first order conditioned stimulus (CS1) and the US-signal stimulus (CSf) were a $1000-\mathrm{Hz}$ continuous tone $(87 \mathrm{~dB})$ and a white noise $(88 \mathrm{~dB})$ from the speaker, counterbalanced across subjects. Each CS was $5 \mathrm{~s}$ in duration.

\section{Procedure}

Sessions were conducted once a day. In the first 12 sessions baseline acquisition training was conducted; rats were allowed to drink from the spout for $300 \mathrm{~s}$ with no event in the CTX-W in each session. They were then divided into three groups (B-P, B-UP, and TRC-P) of 12 rats matched for the number of licks and the body weights of the last three sessions. The characters before the hyphen designate a relationship between the CS1 and the US in the first order conditioning phase: those after hyphen designate a relationship between the CS2 and the CS1 in the second order conditioning phase. The designations B and TRC refer to a relationship 
between CS1 and US: B means backward pairings of US and CS1(US $\rightarrow \mathrm{CS} 1)$, TRC means random presentations of CS1 and US. The designations $\mathrm{P}$ and UP refer to the relationship between CS2 and CS1: $\mathrm{P}$ means paired presentations of CS2 and CS1 $(\mathrm{CS} 2 \rightarrow \mathrm{CS} 1)$ and UP means unpaired presentations of them. The Group B-P was an experimental group on which the second order conditioning procedure was conducted; other two Groups were control groups (Rescorla, 1980).

During the first order conditioning phase, eight conditioning sessions and eight baseline recovery sessions (equivalent to baseline acquisition sessions) are conducted by turn. All subjects were exposed to $20 \mathrm{USs}, 20 \mathrm{CSfs}$, and 20 CS1s in the CTX-B in each conditioning session (1780 $\mathrm{s}$ in duration). The offset of each CSf was followed immediately by the onset of each US. The offset of the US was followed immediately by the onset of each CS1 in Groups B-P and B-UP; in Group TRC-P, the CS1 and the US were arranged randomly (the truly random control procedure; Rescorla, 1967). The mean ITI was 60 $\mathrm{s}$ in Groups B-P and B-UP; 30s in Group TRC-P.

During the second order conditioning phase, six conditioning sessions and six baseline recovery sessions were conducted by turn. All groups were exposed to two CS2s and two CS1s in the CTX-W in each conditioning session (300 $\mathrm{s}$ in duration). The offset of each CS2 was followed immediately by the onset of the CS1 in Groups B-P and TRC-P. In Group B-UP, the CS2 was presented at the same timing as in other two groups, but the CS1 were not paired (the explicitly unpaired procedure). The mean ITI was $100 \mathrm{~s}$ in Groups B-P and TRC-P; $50 \mathrm{~s}$ in Group B-UP.

After the second order conditioning phase, testing for the CS1 and the CSf was conducted for a session of $300 \mathrm{~s}$. Two CS1 and two CSf were presented without the US in the CTX-W, with an average ITI of $75 \mathrm{~s}$.

\section{Results}

Some accident happened in the fifth day in the second order conditioning phase, and licking baseline of each rat was so damaged that the data on that day was omitted from the analysis. In all statistical analyses reported in this article, the criterion for rejection of the null hypothesis was .05 .

Figure 1 shows the suppression to CS2 in the second order conditioning phase in terms of suppression ratios (SR; cf. Annau \& Kamin, 1961) for each conditioning day. Each data point was calculated by dividing the total number of licks during CS2s in a session by the sum of it and the total number of licks during $5 \mathrm{~s}$ pretrial periods of the same session. A 3 (groups) X 5 (sessions) ANOVA yielded significant main effects of groups, $F(2,33)=10.16$, and sessions, $F(4,132)=$ 10.17. Their interaction was not significant, $F(8$, $132)=1.69$. Fisher's $L S D$ tests revealed that both control groups, B-UP and TRC-P, showed less suppression to the CS2 than B-P, but they did not differ significantly from each other. Though the interaction was not significant, in order to inspect the process of forming the second order excitation, Fisher's $L S D$ tests were conducted among groups in each session. As the result, differences between Groups B-P and B-UP were significant in sessions 2, 3, 4, and 6, differences between Groups B-P and TRC-P were significant in sessions 2, 3, and 4, and differences between Groups TRC-P and B-UP were significant in sessions 2,3 , and 6 .

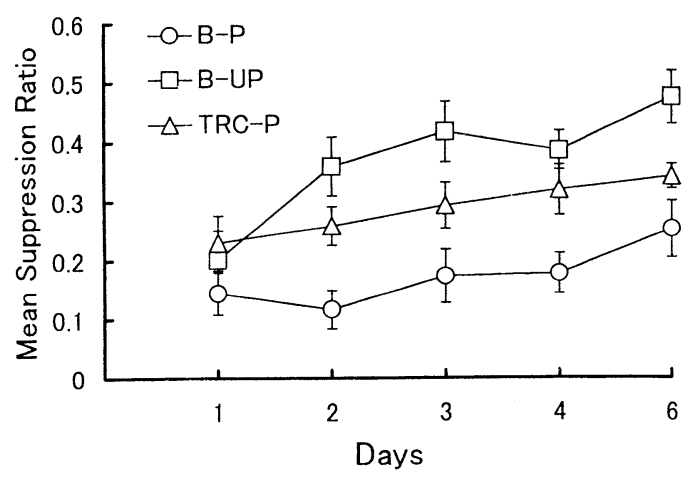

Figure 1. Mean $( \pm S E M)$ suppression ratios of licking to the CS2 during second order conditioning phase. The CS2 was a diffuse light in each group.

The testing data of CS1 and CSf are shown in Figure 2. The SR was calculated by dividing the total number of licks during CSs in a session by the sum of it and the total number of licks during $5 \mathrm{~s}$ pretrial periods of the same session. Subjects 
in all groups showed strong suppressions to CSf but little suppression to CS1; no significant differences were seen among groups (to CS1, $F(2$, $33)=1.44$; to CSf, $F<1$ ).

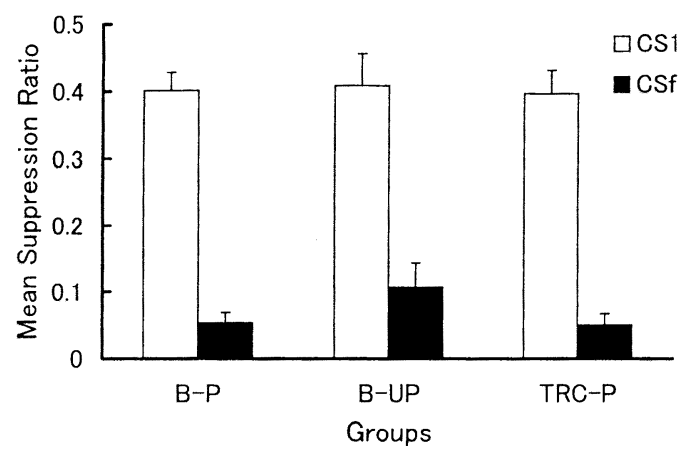

Figure 2. Mean (+SEM) suppression ratios of licking to the CS1 and the CSf during test phase. The CS1 and the CSf were a continuous tone and a noise, counterbalanced.

\section{Discussion}

Subjects in Group B-P, the experimental second order conditioning group, showed greater suppressions to the CS2 in the second order conditioning phase, whereas no significant suppression to CS1 was observed in the later test session. It seems to be a successful double check of previous studies. It appears to be demonstrate the second order excitation with first order backward conditioning observed in Barnet and Miller (1996) and Cole and Miller (1999). The result of the present experiment would indicate that the backward conditioned CS1 had the power to establish an excitatory second order conditioning, while it showed little or no power to elicit excitatory CRs to itself.

In addition, we successfully observed the acquisition process of the second order excitation with backward first order conditioning. All groups, including experimental and control groups, showed moderate suppression at the first session, but only the suppression in Group B-P. remained relatively long, while those in the other two control groups vanished rapidly. The suppressions observed at the first session in all group were thought to be a kind of sensitization caused by the exposure to so many strong USs.

This phenomenon, the second order excitation with first order backward conditioning, is similar to the effects observed in Barnet and Miller (1996) and Cole and Miller (1999). On the other hand, the result is inconsistent with that of Urushihara and Imada (1999), though their experiment was conducted with almost the same apparatus and procedure as this experiment. This discrepancy between the results in the two experiments may result from the difference in the number of the first order backward conditioning trials (24 in the Urushihara and Imada, 160 in the present experiment), as indicated by Cole and Miller (1999), but the comparison between these studies never tells enough. Further studies are needed to form a conclusion about this issue.

\section{References}

Annau, Z., \& Kamin, L. T. 1961 The conditioned emotional response as a function of intensity of the US. Journal of Comparative and Physiological Psychology, 51, 128-132.

Barnet, R. C., \& Miller, R. R. 1996 Second-order conditioning mediated by a backward conditioned inhibitor. Journal of Experimental Psychology: Animal Behavior Processes, 22, 279-296.

Cole, R. P., \& Miller, R. R. 1999 Conditioned excitation and conditioned inhibition acquired through backward conditioning. Learning and Motivation, 30, 129-156.

Hemmes, N. S., Brown, B. L., \& De Vaca, C. S. 1994 Appetitive backward conditioning in pigeons. The Psychological Record, 44, 221-237.

Matzel, L. D., Held, F. P., \& Miller, R. R. 1988 Information and expression of simultaneous and backward associations: Implications for contiguity theory. Learning and Motivation, 19, 317-344.

Pavlov, I. P. 1927 Conditioned reflexes: An investigation of the physiological activities of the cerebral cortex. London: Oxford University Press.

Rescorla, R. A. 1967 Pavlovian conditioning and its proper control procedure. Journal of Comparative and Physiological Psychology, 64, 114-120.

Rescorla, R. A. 1980 Pavlovian second-order 
conditioning. Hillsdale, NJ: Erlbaum.

Stern, S. D., \& Frey, P. W. 1978 Backward conditioning of the rabbit eyelid response: A test using second-order conditioning. Bulletin of the Psychonomic Society, 11, 231234.

Urushihara, K. 1999 Recent studies on classical backward conditioning. Japanese Psychological Review, 42, 272-286.

Urushihara, K., \& Nakajima, S. 1999 Temporal coding hypothesis in Pavlovian conditioning. Journal of the Literary Association of Kwansei Gakuin University, 48, 29-40.

Urushihara, K., \& Imada, H. 1999 Effect of temporal relationship between the first-order conditioned stimulus and the unconditioned stimulus upon classical second-order conditioning in rats. Japanese Journal of Animal psychology, 49, 161-170.

(Received Oct. 6, 1999 ; accepted Feb. 26, 2000) 